\title{
Quantum-mechanical Rotational and Vibrational Signatures of Astrophyscially relevant Gas-Phase Stereo-isomeric Species of Leucine
}

\author{
Namrata Rani ${ }^{1}$ and . Vikas $^{1}$ \\ ${ }^{1}$ Panjab University
}

December 22, 2020

\begin{abstract}
The search for life-supporting molecules in outer space is an ever growing endeavour. Towards this, the quantum-mechanical computations supporting the astronomical spectroscopic observations are becoming valuable tools to unravel the complex chemical network in the interstellar medium (ISM). In the present work, quantum-mechanical computations are performed to obtain the rotational and vibrational line-data of gas-phase conformers of amino acid Leucine and its isomeric species predicted to be involved in its stereoinversion under the conditions of ISM. These species exhibit diverse chemistry including branched skeleton and zwitterionic ammonium ylides. Notably, the present work employs vibrational second order perturbation theory to account for anharmonic effects in rotational and vibrational transitions. The spectroscopic data computed in this work can assist in the detection of Leucine and its isomeric species in different regions of ISM.
\end{abstract}

Quantum-mechanical Rotational and Vibrational Signatures of Astrophyscially relevant GasPhase Stereo-isomeric Species of Leucine

Namrata Rani and Vikas*

Quantum Chemistry Group, Department of Chemistry \&3 Centre of Advanced Studies in Chemistry, Panjab University, Chandigarh-160014, India.

*Corresponding author e-mail: qlabspu@pu.ac.in, Tel: +91-172-2534408

\section{Abstract}

The search for life-supporting molecules in outer space is an ever growing endeavour. Towards this, the quantum-mechanical computations supporting the astronomical spectroscopic observations are becoming valuable tools to unravel the complex chemical network in the interstellar medium (ISM). In the present work, quantum-mechanical computations are performed to obtain the rotational and vibrational line-data of gas-phase conformers of amino acid Leucine and its isomeric species predicted to be involved in its stereoinversion under the conditions of ISM. These species exhibit diverse chemistry including branched skeleton and zwitterionic ammonium ylides. Notably, the present work employs vibrational second order perturbation theory to account for anharmonic effects in rotational and vibrational transitions. The spectroscopic data computed in this work can assist in the detection of Leucine and its isomeric species in different regions of ISM.

Keywords: Astrochemistry; spectroscopy; quantum chemistry; amino acids; anharmonic effects.

\section{INTRODUCTION}


Spectroscopy is the only viable route to probe exotic species in the challenging environment of interstellar medium (ISM) ${ }^{1,2}$ In fact, numerous anomalous species are being reported in the ISM, ${ }^{3,4}$ for example, the molecular ions of noble gases such as $\mathrm{ArH}^{+}$and $\mathrm{HeH}^{+}$, the equal abundance of $\mathrm{HCN}$ and its terrestrially less stable isomer $\mathrm{HNC}$, significant abundance of branched chain isopropyl cyanide compared to its linear chain isomer, to name a few. ${ }^{5-7}$ To interpret and resolve the complex spectroscopic information received from the molecular species present in the ISM, the insights from quantum-mechanical computations have been becoming quite valuable, ${ }^{8}$ which in fact has played quite a significant role in updating the interstellar census of molecules. ${ }^{9}$ In our recent study,${ }^{10}$ the stereoinversion of Leucine, under gas-phase conditions akin to ISM, was revealed to proceed through exotic molecular species which exhibits diverse chemistry (see later).

The rotational spectroscopy has remained the key method for remote observation of interstellar and circumstellar species. The spectroscopy in millimetre $(\mathrm{mm})$ and sub-mm regions of wavelength corresponds to rotational transitions. In fact, it is the rotational spectroscopy which has led to the detection of majority of the species identified in the outer space. ${ }^{11,12}$ The structure specific characteristics of rotational transitions can be used to differentiate the molecular species irrespective of having the same mass. Moreover, this has also been responsible for driving the advancement in the futuristic telescopes in $\mathrm{mm} / \mathrm{sub}-\mathrm{mm}$ region. ${ }^{13}$ However, the laboratory based studies on interstellar ices are generally carried out in the infrared (IR) region with wavelengths lower than sub-mm. ${ }^{14}$ Now-a-days, the IR spectral predictions via quantum computations are widely assisting such laboratory studies. In fact, the confirmation of molecular species such ascyclo $-\mathrm{C}_{3} \mathrm{H}_{2}, \mathrm{HOCO}^{+}$, HSS etc. ${ }^{15-20}$ in the ISM are exemplary towards such assistance by quantum computational spectroscopy to the laboratory IR spectra and hence the astronomical observations. Therefore, the spectroscopic data computed in the rotational and infrared regions is quite valuable in sorting the complex spectroscopic information received from the outer space as well as from the laboratories mimicking the outer space environment. ${ }^{21}$ Not only this, the interstellar spectroscopic studies are providing unique opportunities to broaden up our understanding of physical and chemical characteristics of the universe. ${ }^{22,23}$ The various regions of ISM are speculated to be associated with all the core functional groups of organic prominence, thereby, acting as significant players in the extra-terrestrial formation of bio-molecules. At present, it is quite clear that ISM is home to branched chain molecules and chiral molecules but the specificity in their handedness is still debated. ${ }^{24-25}$

In the present work, the rotational and vibrational signatures are quantum-mechanically computed for the important conformers of Leucine and its isomers responsible for its gas-phase stereoinversion under the conditions of ISM as revealed in our previous study. ${ }^{10}$ Leucine is the most abundant amino acid in the proteins, therefore, it is reasonable to advocate prioritizing its search in the ISM relative to other proteinogenic amino acids. Moreover, the branched chain amino acids found in meteoritic composition dominate over the straight chain isomers, which additionally acts as a proponent for the search of Leucine in outer space. ${ }^{27}$ The ISM is a region of isolated molecules on account of ultra-low pressure and density. ${ }^{28}$ Thus, its molecular characterization involves the gas-phase spectral analysis without solvent consideration. In the existing literature, a gas-phase rotational study has been performed by Cocinero et al. ${ }^{29}$ to identify the conformers of Leucine through Fourier transform laser-ablation microwave experiments. Besides this, two different experimental studies are also available on the gas-phase vibrational analysis of Leucine for characterizing its conformers. The recent of the two studies is based on low-temperature matrix-isolation IR experiments by Stepanian et al. ${ }^{30}$ However, the second study probing the IR spectra is based on a high temperature fast thermal heating experiments by Linder et al. ${ }^{31}$ Additionally, the latter analysis also signifies the stability of amino acids under high temperature of up to $570 \mathrm{~K}$ which supports the presence of Leucine in the hot cores of the protostars in the ISM. In fact, this region of ISM has been proposed as a suitable region for observing stereoinversion in Leucine. ${ }^{10}$ Apart from the experimental studies, anab-initio investigation of rotational and vibrational spectra of gas-phase conformers of Leucine has also been reported by Dokmaisrijan et al. ${ }^{32}$

In the present work, the rotational and vibrational parameters have been computed for the conformers of Leucine as well as its stereo-isomeric species (depicted in Figure 1) using the methodology described in the next section. The species studied include global minimum conformer of Leucine $\left(\mathrm{EQ} 0_{\#}\right)$, its conformers $\left(\mathrm{EQ0}_{\mathrm{I}}, \mathrm{EQ0}_{\mathrm{II}}, \mathrm{EQ0}_{\mathrm{III}}, \mathrm{EQ0}_{\mathrm{IV}}\right)$ besides the isomeric species explored along the gas-phase stereoinversion path- 
ways of Leucine. ${ }^{10}$ Notably, the isomeric species investigated have diverse chemistry involving zwitterionic ammonium ylides (EQ1 $\left.1_{\mathrm{R} 1}, \mathrm{EQ} 1_{\mathrm{R} 3 \mathrm{a}}, \mathrm{EQ} 2_{\mathrm{R} 3}\right)$, imine-diol (EQ1 $2 \mathrm{R}$, $\left.\mathrm{EQ} 2_{\mathrm{R} 2 \mathrm{a}}, \mathrm{EQ} 1_{\mathrm{R} 4}\right)$, and ene-diol (EQ1 $\left.1_{\mathrm{R} 2 \mathrm{~b}}\right)$ besides having a branched carbon-chain skeleton. In fact, ammonium ylides are known to be involved in the synthesis of amino acids. The computed spectroscopic parameters of the molecular species investigated in this work are further compared with available experimental data besides emphasizing the astrophysical importance of the data as discussed in the subsequent sections.

\section{Hosted file}

image1. emf available at https://authorea.com/users/384438/articles/500049-quantum-mechanicalrotational-and-vibrational-signatures-of-astrophyscially-relevant-gas-phase-stereoisomeric-species-of-leucine

Figure 1. The conformers $\left(\mathrm{EQ}_{\#}, \mathrm{EQ} 0_{\mathrm{I}}, \mathrm{EQ} 0_{\mathrm{II}}, \mathrm{EQ} 0_{\mathrm{III}}, \mathrm{EQ} 0_{\mathrm{IV}}\right)$ of Leucine and stereo-isomeric species $\left(\mathrm{EQ}_{\mathrm{R} 1}, \mathrm{EQ} 1_{\mathrm{R} 2 \mathrm{a}}, \mathrm{EQ} 2_{\mathrm{R} 2 \mathrm{a}}, \mathrm{EQ} 1_{\mathrm{R} 2 \mathrm{~b}}, \mathrm{EQ} 1_{\mathrm{R} 3 \mathrm{a}}, \mathrm{EQ} 2_{\mathrm{R} 3}, \mathrm{EQ} 1_{\mathrm{R} 4}\right)$ predicted along the gas-phase stereoinversion pathways of Leucine [Ref. 10]. The labelling of various species corresponds to the pathways (for details see Ref. 10) The geometries depicted are optimized at DFT/B3LYP-D3(BJ)/cc-pVTZ level of the theory. The dashed line represents the hydrogen bonding interactions (with atomic distances in $\AA$ ).

\section{COMPUTATIONAL METHODOLOGY}

The methodology adopted in the present work to compute the rotational and vibrational data is similar to that utilized for the spectral study of Glutamic acid in our previous such work. ${ }^{33}$ The conformers of biologically significant left-handed isomer $(L)$-Leucine and its isomeric species being investigated in this work are depicted in Figure 1. Note that the global minimum (lowest-energy conformer) of Leucine (EQ0\#) has been verified through its structure optimization performed at different levels of quantum-mechanical theory (for details, see Ref. 10). For the present work, ab-initio Møller-Plesset $2^{\text {nd }}$ order (MP2) perturbation theory with frozen-core (fc) approximation as well as density functional theory (DFT) are utilized to compute the spectroscopic parameters. ${ }^{34-35}$ The rotational constants computed for EQ0\# at different levels of the theory are provided in Table 1. These are also compared with the available experimental gas-phase data from a study by Cocinero et al. ${ }^{29}$ The computed rotational constants are found to be in good agreement with experimental one. In particular, the MP2 $/ 6-31+\mathrm{G}(\mathrm{d}, \mathrm{p})$ method using a Gaussian basis set, $6-31+\mathrm{G}(\mathrm{d}, \mathrm{p})$, resulted in a more closer agreement with deviations less than $5 \mathrm{MHz}$ compared to MP2/6-311++G(d,p) method exhibiting deviations up to $7 \mathrm{MHz}$, as evident in Table 1. This observation in the present work, thereby, improves upon the previously known computational results of Cocinero et al., ${ }^{29}$ in their prediction of rotational parameters through MP2/6-311++G(d,p) method which was used to assist their experimental results.

Besides the aforementioned wave-function based MP2 method, the DFT computations for rotational constants were also carried out. For this, a conventional hybrid exchange correlational functional, B3LYP, is employed along with a correlation consistent, cc-pVTZ and aug-cc-pVTZ, basis set. ${ }^{37,38}$ Additionally, in view of various non-covalent (dispersion) interactions in the branched structural framework of Leucine, Grimme's D3 dispersion correction has also been employed along with a Becke-Johnson (BJ) damping function in the computaions. ${ }^{39,40}$ It is quite evident from Table 1 that DFT/B3LYP-D3(BJ)/cc-pVTZ method gives more closer values (to the experimental one) for rotational constants of global conformer, with deviation of only $5 \mathrm{MHz}$ than that computed using the DFT/B3LYP-D3(BJ)/aug-cc-pVTZ method. Besides this, a recently proposed SNSD basis set, ${ }^{41}$ constituted from the polarized double-zeta $(\zeta)$ N07D basis set including the diffuse s-typefunctions on every atom and diffuse polarized (d-type) functions on heavy atoms as well as p-type on hydrogen atoms, is also tested in besides the B3LYP-D3(BJ) method. However, though SNSD is known to be quite accurate and cost-effective for spectroscopic calculations but in the present work, it is observed to be less accurate than B3LYP-D3(BJ)/cc-pVTZ method. Moreover, in the present work, DFT calculations were also performed employing a recently recommended double hybrid functional, B2PLYPD3(BJ) ${ }^{42}$ however, significant deviations of more than $10 \mathrm{MHz}$ are observed using this method as can be seen in Table 1. Thus, overall, B3LYP-D3(BJ)/cc-pVTZ is observed to be the best DFT method although 
it is found to be relatively less accurate than the wave-function based $a b$ initioMP2/6-31+G(d,p) method as clearly evident in Table 1.

On the basis of aforementioned observations for the global conformer, the rotational parameters of other isomeric species being investigated in this work were computed using their optimized geometries at both the aforementioned quantum-mechanical levels of theory, namely, MP2/6-31+G(d,p) and DFT/B3LYPD3(BJ)/cc-pVTZ. The optimized structures were additionally validated through harmonic vibrational frequency check. However, anharmonic frequency calculations are also performed at the specified levels of the theory to accurately estimate the vibrational frequencies beyond harmonic oscillator approximation, which further provided other rotational parameters listed in Table 2, for example, the Ray's asymmetry parameter, quartic centrifugal distortion constants, and nuclear quadrupole coupling constants. Note that for these calculations, a quartic force field (QFF) is deployed for the inter-nuclear potential in the 'Watson' Hamiltonian so as to account for ro-vibrational interactions. ${ }^{9,21}$ Further, for the computation of vibrational frequencies, the anharmonicity is estimated at the level of the second order vibrational perturbation theory (VPT2) which is basically rooted in the Rayleigh-Schrödinger perturbation theory. ${ }^{43,44}$ Note that the inclusion of anharmonic effects actually contribute towards an accurate estimation of both vibrational and rotational parameters, ${ }^{21}$ by aiding the evaluation of rotational constants associated with vibrationally averaged ground state. Besides this, the anharmonic calculations also provide the centrifugal distortion constants which are estimated employing Watson's A-reduced Hamiltonian in irreducible $\left(\mathrm{I}^{\mathrm{r}}\right)$ representation. ${ }^{45,46}$

In the present work, all the aforementioned quantum-mechanical computations are performed using Gaussian 09 program. ${ }^{47}$ The rotational parameters computed at MP2 $/ 6-31+\mathrm{G}(\mathrm{d}, \mathrm{p})$ level of the theory, as collected in Tables 2 and 3, were further used for generating the rotational spectral line data and simulating the rotational spectra through the PGOPHER program ${ }^{48}$ as depicted in Figure 2 as well as listed in the Supporting Information (SI) Tables S1 and S2. Besides this, the anharmonic vibrational spectroscopic data computed for the global minimum conformer EQ0 \# is further compared in Table 4 with the experimental gas-phase values for Leucine available from matrix-IR and fast thermal heating experiments. ${ }^{30,31}$ As evident in Table 4, DFT/B3LYP-D3(BJ)/cc-pVTZ exhibits a closer agreement with the experiment. Thus, this DFT method was employed for computing the vibrational spectral data for all other species in the complete IR region (from mid-IR to far-IR). Additionally, a VEDA4 program, ${ }^{49}$ has also been used to further characterize each mode of vibration in terms of potential energy distribution (PED) for all the conformers of Leucine and its isomeric species, as provided in supporting information SI Table S3. It should be noted that in the present work, these calculations are performed at a temperature of $298.15 \mathrm{~K}$, the effect of temperature on vibrational lines is not attempted in this work. However, since the rotational intensities considerably depend on the temperature, therefore, while simulating the rotational spectra and generating the line-data through PGOPHER program, ${ }^{45}$ a low temperature of $10 \mathrm{~K}$ is used in order to assist the search of species in cold molecular clouds as elaborated in the next section.

Table 1. Computed equilibrium rotational constants $\left(A_{e}, B_{e}, C_{e}\right)$ for the global minimum conformer of Leucine $\left(\mathrm{EQ} 0_{\#}\right)$ at various levels of quantum-mechanical (MP2 and DFT) methods. The respective absolute deviations relative to the experimental values are listed in parenthesis. The best estimated values are marked in bold, for details see the text.

\begin{tabular}{llll}
\hline Method & $\mathbf{A}_{\mathbf{e}}(\mathbf{M H z})$ & $\mathbf{B}_{\mathbf{e}}(\mathbf{M H z})$ & $\mathbf{C}_{\mathbf{e}}(\mathbf{M H z})$ \\
\hline Experimental [Ref. 29] & 2753.17 & 846.65 & 794.61 \\
MP2/6-31+G(d,p) & $\mathbf{2 7 5 4 . 3 1 ( 1 . 1 4 )}$ & $\mathbf{8 4 9 . 3 6 ( 2 . 7 )}$ & $\mathbf{7 9 9 . 5 7 ( 5 . 0 )}$ \\
MP2/6-311++G(d,p) & $2756.20(3.03)$ & $852.96(6.3)$ & $801.93(7.3)$ \\
{$[$ Present work] } & & 853 & 802 \\
MP2/6-311++G(d,p) & 2756 & & $\mathbf{7 9 6 . 8 0 ( 2 . 2 )}$ \\
[Ref. 29] & & $\mathbf{8 4 3 . 0 9 ( - 3 . 6 )}$ & \\
DFT/B3LYP- & $\mathbf{2 7 4 8 . 0 6 ( - 5 . 1 1 )}$ & &
\end{tabular}




\begin{tabular}{llll}
\hline Method & $\mathbf{A}_{\mathbf{e}}(\mathbf{M H z})$ & $\mathbf{B}_{\mathbf{e}}(\mathbf{M H z})$ & $\mathbf{C}_{\mathbf{e}}(\mathbf{M H z})$ \\
\hline $\begin{array}{l}\text { DFT/B3LYP- } \\
\text { D3(BJ)/aug-cc-pVTZ }\end{array}$ & $2735.74(-17.43)$ & $843.18(-3.5)$ & $797.19(2.6)$ \\
$\begin{array}{l}\text { DFT/B3LYP- } \\
\text { D3(BJ)/SNSD }\end{array}$ & $2715.24(-37.93)$ & $828.90(-17.7)$ & $774.66(-20.0)$ \\
DFT/B2PLYP- & $2766.67(13.50)$ & $849.40(2.8)$ & $800.67(6.1)$ \\
D3(BJ)/cc-pVTZ & & $844.39(2.3)$ & $796.95(6.6)$ \\
DFT/B2PLYP- & $2739.14(-14.03)$ & & \\
D3(BJ)/SNSD & & &
\end{tabular}

Table 2. Rotational parameters, computed at the MP2/6-31+G(d,p) level of theory, for all the species depicted in Figure 1. $\mathrm{A}_{\mathrm{e}}, \mathrm{B}_{\mathrm{e}}, \mathrm{C}_{\mathrm{e}}$ are the equilibrium rotational constants (in $\mathrm{MHz}$ ), $\mathrm{A}_{0}, \mathrm{~B}_{0}, \mathrm{C}_{0}$ are vibrationally corrected rotational constants (in MHz), $x$ is the Ray's asymmetry parameter, $\Delta_{\mathrm{J}}, \Delta_{\mathrm{K}}, \Delta_{\mathrm{JK}}, \delta_{\mathrm{J}}, \delta_{\mathrm{K}}$ are the quartic centrifugal distortion constants (in $\mathrm{kHz}$ ), $\chi_{\mathrm{aa}}, \chi_{\mathrm{bb}}, \chi_{\mathrm{cc}}$ are the nuclear quadrupole coupling constants (in $\mathrm{MHz}$ ), $\mu$ is the dipole moment (in Debye), all calculated at MP2/6-31+G(d,p) level of theory. (The values of parameters were obtained using Watson's A-reduced asymmetric top Hamiltonian in $\mathrm{I}^{\mathrm{r}}$ representation).

\begin{tabular}{|c|c|c|c|c|c|c|c|c|c|c|c|}
\hline & $\mathrm{EQ0}_{\#}$ & EQ0 $_{\text {I }}$ & $\mathrm{EQ0}_{\text {II }}$ & EQ0 $_{\text {III }}$ & $\mathrm{EQ0}_{\mathrm{IV}}$ & $\mathrm{EQ1}_{\mathrm{R} \mathbf{1}}$ & EQ1 $_{R 2 a}$ & $\mathrm{EQ2}_{\mathrm{R} 2 \mathrm{a}}$ & $\mathrm{EQ1}_{\mathrm{R} 2 \mathrm{~b}}$ & EQ1 $_{R 3 a}$ & $\mathrm{EQ2}_{\mathrm{R} 3}$ \\
\hline$A_{\varepsilon}$ & 2754.3 & 2864.1 & 1951.3 & 2847.2 & 1948.0 & 2473.3 & 2588.2 & 3026.4 & 2299.6 & 3938.6 & 3712.2 \\
\hline $\mathrm{B}_{\varepsilon}$ & 849.4 & 911.5 & 1365.0 & 920.9 & 1362.9 & 1053.6 & 1075.1 & 866.6 & 1153.8 & 640.8 & 737.5 \\
\hline $\mathrm{C}_{\mathrm{e}}$ & 799.6 & 822.4 & 1124.9 & 814.5 & 1116.8 & 839.6 & 892.7 & 804.6 & 904.0 & 624.0 & 667.0 \\
\hline $\mathbf{A}_{0}$ & 2729.4 & 2834.0 & 1943.4 & 2817.6 & 1938.1 & 2420.9 & 2585.2 & 2997.5 & 2284.4 & 4065.7 & 3714.8 \\
\hline $\mathbf{B}_{0}$ & 841.8 & 902.8 & 1340.3 & 912.3 & 1339.3 & 1056.5 & 1056.4 & 859.4 & 1138.9 & 618.9 & 722.6 \\
\hline $\mathrm{C}_{0}$ & 791.4 & 814.1 & 1104.5 & 805.2 & 1097.5 & 841.0 & 879.0 & 798.2 & 893.0 & 607.3 & 654.6 \\
\hline$x$ & -0.95 & -0.91 & -0.42 & -0.90 & -0.41 & -0.74 & -0.78 & -0.94 & -0.64 & -0.99 & -0.95 \\
\hline$\Delta_{\Theta}$ & 0.06 & 0.06 & 0.36 & 0.06 & 0.36 & 0.24 & 0.23 & 0.06 & 0.22 & 0.09 & 0.12 \\
\hline$\Delta_{\mathrm{K}}$ & 0.66 & 0.15 & 0.49 & 0.37 & 0.51 & 1.95 & 2.58 & -1.73 & 0.73 & 6.86 & 4.13 \\
\hline$\Delta_{\Theta \mathrm{K}}$ & 0.31 & 0.43 & -0.36 & 0.25 & -0.39 & -0.81 & -0.69 & 2.01 & -0.21 & -0.66 & -0.59 \\
\hline$\delta_{\Theta}$ & 0.00 & 0.00 & 0.08 & 0.01 & 0.08 & 0.05 & 0.05 & 0.01 & 0.05 & 0.01 & 0.01 \\
\hline$\delta_{K}$ & 0.79 & 0.36 & 0.16 & 0.44 & 0.13 & 0.09 & 0.45 & -1.23 & 0.29 & 0.80 & 0.17 \\
\hline$\chi_{\alpha \alpha}$ & -3.47 & -3.94 & -3.47 & -3.92 & -0.53 & -0.20 & -1.52 & -1.36 & -2.80 & 0.31 & 0.31 \\
\hline$\chi_{\beta \beta}$ & 2.02 & 2.47 & 1.63 & 2.58 & 0.58 & -0.06 & 0.87 & 0.10 & 2.43 & 0.34 & -0.87 \\
\hline$X_{55}$ & 1.45 & 1.47 & 1.89 & 1.34 & -0.04 & 0.26 & 0.65 & 1.27 & 0.37 & -0.65 & 0.56 \\
\hline$\mu_{a}$ & 0.05 & 0.31 & -1.71 & 0.26 & -0.33 & 1.38 & 3.52 & -2.50 & 0.45 & 6.22 & 6.11 \\
\hline$\mu_{\mathrm{b}}$ & -0.89 & 1.45 & -0.96 & -1.17 & -1.00 & 7.03 & -0.71 & 0.97 & 1.21 & 1.11 & -1.49 \\
\hline$\mu_{c}$ & 0.52 & -0.49 & 1.39 & -0.02 & -0.77 & 0.06 & 0.51 & -1.03 & 0.37 & -0.73 & 0.35 \\
\hline$\mu$ & 1.03 & 1.56 & 2.40 & 1.20 & 1.30 & 7.16 & 3.63 & 2.87 & 1.34 & 6.36 & 6.30 \\
\hline
\end{tabular}

\section{RESULTS AND DISCUSSIONS}

\subsection{Rotational Analysis}

There are basically two approaches to analyse the rotational spectra: 'Bottom Up' and 'Top Down'. The first approach utilizes the computed rotational parameters to simulate the spectra whereas the other approach involves the estimation of rotational parameters from the observed rotational spectra. Quantum-mechanical calculations for computing rotational spectroscopic parameters had already manifested their proficiency in assisting the experimental investigations. ${ }^{50}$ In case of astronomical detection of molecular species, the quantummechanically computed rotational parameters are being utilized through different working strategies. ${ }^{51}$

A 'Bottom Up' approach is generally used if an experimental laboratory based rotational study on the 
molecular species is either not available or cannot be carried out. In such a case, the simulated rotational spectrum computed quantum-mechanically can be directly compared with the astronomical observations. This strategy becomes extremely important particularly in the case of charged and radical species because such species are difficult to detect due to their tendency to form stable terrestrial molecules by reacting with the similar reactive species. In fact, the astrophysical detection of $\mathrm{C}_{5} \mathrm{~N}^{-}$ion and $\mathrm{C}_{4} \mathrm{H}$ radical was entirely based on the quantum mechanical computations. ${ }^{52-54}$

On the contrary, in the 'Top Down' approach, a laboratory based experimental rotational study is an essential pre-requisite so as to fit the rotational spectra obtained from experiments with the simulated rotational spectrum from quantum calculations. The fitting will thus provide the experimental rotational parameters which can be used to assist the astronomical observations. This is the most conventional approach to detect cosmic species in a definitive manner. The recent detection of first disilicon molecule, SiCSi, in the ISM is the outcome of this kind of synergic working module between astronomical observatories, laboratory experiments, and quantum mechanical computations. ${ }^{55,56}$

However, the present study employs an additional strategy for improving the computed rotational parameters. In this approach, the computed rotational parameters, particularly the rotational constants, are appropriately scaled for the species for which no experimental data is available. For this, a scaling factor is determined using the experimentally known rotational parameters of a similar reference species studied in the literature. ${ }^{57}$ This strategy is in fact parallel to the 'Bottom Up' approach but employing scaled and more accurate rotational parameters. In the present work, the gas-phase experimental rotational data is available for global minimum conformer $\mathrm{EQ} 0_{\#}$ of Leucine, thereby acting as the reference molecular species. The experimental rotational constants of this reference species was then used to determine a suitable scaling factor to evaluate the rotational constants of other conformers of Leucine and isomeric species for which no experimental rotational data is available. The scaled rotational constants so obtained were further used to simulate the rotational spectra in rotational frequency region of interest as discussed below.

As analysed in the previous section, MP2/6-31+G(d,p) was found to be the optimal method for rotational calculations. For the global conformer EQ0\#, the spectrum simulated using the computed rotational constants is compared with that simulated using experimental rotational constants in Figure 2. Note that only ' $\mathrm{R}$ ' branch transitions (following the selection rule $\Delta J=+1$, in terms of principal rotational quantum number $J$ ) are considered because of their much higher intensity compared to ' $\mathrm{Q}$ ' branch $(\Delta J=0)$ and 'P' branch $(\Delta J=-1)$ transitions. For simplicity, the spectrum has been simulated in the lower frequency range of $4000 \mathrm{MHz}$ to $20000 \mathrm{MHz}$, as applicable to the laboratory observations, ${ }^{29}$ while limiting the value of principal rotational number to $J=5$. A detailed comparison of rotational line data predicted from the computed parameters with those from the experimental parameters ${ }^{29}$ is further provided in SI Table S1. Nevertheless, from Figure 2, it is apparent that both the spectra follow the same pattern of rotational transitions. A closer observation, however, indicates some deviation in the position of rotational lines which can be as small as $8 \mathrm{MHz}$ involving lower rotational states, for example, $J$ (2 1) transition. The deviation, however, continuously increases with higher rotational states. Further, the transitions corresponding to the lower value of rotational quantum numbers correspond to the low frequency region. However, the telescopic observations of rotational transitions are generally studied in higher frequency region than the frequency range involved in laboratory studies. ${ }^{13}$ The deviation in the rotational line location may increase even more for higher frequency regions as can be observed from SI Table S1. Moreover, a notable difference of 65 $\mathrm{MHz}$ (as evident in SI Table S1) is not acceptable if a direct comparison with the telescopic observations is required. Therefore, in the present work, a scaling factor becomes inevitable. The procedure adopted for obtaining the scaling factors is similar to the one employed in our previous study on Glutamic acid. ${ }^{33}$ In the present work, the ratio of experimental (exp) and computed rotational constants of global conformer EQ0\# act as scaling factors, thus, $0.999\left(\mathrm{~A}_{\exp } / \mathrm{A}_{\mathrm{e}}\right), 0.996\left(\mathrm{~B}_{\exp } / \mathrm{B}_{\mathrm{e}}\right), 0.994\left(\mathrm{C}_{\exp } / \mathrm{C}_{\mathrm{e}}\right)$ are the scaling factor used for the rotational constants $\mathrm{A}, \mathrm{B}$ and $\mathrm{C}$, respectively. The scaled rotational constants calculated for all the species are further listed in Table 3.

\section{Hosted file}


image2 . emf available at https://authorea.com/users/384438/articles/500049-quantum-mechanicalrotational-and-vibrational-signatures-of-astrophyscially-relevant-gas-phase-stereoisomeric-species-of-leucine

Figure 2. Simulated rotational spectra of global conformer EQ0 \# of Leucine: Experimental spectrum (depicted in red) is simulated using the rotational parameters taken from Ref. [29], whereas the computed spectrum (depicted in black) is simulated using rotational parameters computed in the present work (see Table 2). The spectra are simulated in the frequency range of $4000 \mathrm{MHz}$ to $18000 \mathrm{MHz}$ at a temperature of $10 \mathrm{~K}$. For simplicity, hyperfine splitting is not included in the simulated spectra.

Table 3. Scaled rotational constants (in $\mathrm{MHz}$ ) for species depicted in Figure 1. The scaling factors employed are obtained with reference to the known experimental values of EQ0\# (for details, see the text).

\begin{tabular}{llllllllllll}
\hline & $\mathbf{E Q 0}_{\mathbf{I}}$ & $\mathbf{E Q 0}_{\mathbf{I I}}$ & $\mathbf{E Q 0}_{\mathbf{I I I}}$ & $\mathbf{E Q 0}_{\mathbf{I V}}$ & $\mathbf{E Q 1}_{\mathbf{R 1}}$ & $\mathbf{E Q 1}_{\mathbf{R 2 a}}$ & $\mathbf{E Q 2}_{\mathbf{R 2 a}}$ & $\mathbf{E Q 1}_{\mathbf{R 2 b}}$ & $\mathbf{E Q 1}_{\mathbf{R 3 a}}$ & $\mathbf{E Q 2}_{\mathbf{R 3}}$ & EQ1$_{\mathbf{R 4}}$ \\
\hline $\mathbf{A}_{\boldsymbol{\sigma} \boldsymbol{\alpha} \boldsymbol{\alpha \lambda}}$ & 2863.0 & 1950.5 & 2846.0 & 1947.2 & 2472.3 & 2587.1 & 3025.2 & 2298.6 & 3936.9 & 3710.6 & 2779.2 \\
$\mathbf{B}_{\boldsymbol{\sigma} \boldsymbol{\alpha} \boldsymbol{\lambda}}$ & 908.6 & 1360.6 & 918.0 & 1358.6 & 1050.2 & 1071.6 & 863.8 & 1150.2 & 638.8 & 735.2 & 1067.8 \\
$\mathbf{C}_{\text {scal }}$ & 817.3 & 1117.9 & 809.4 & 1109.8 & 834.4 & 887.1 & 799.6 & 898.4 & 620.2 & 662.8 & 949.7 \\
\hline
\end{tabular}

Further, as evident in Table 2, all the species being investigated in this work possess a net non-zero dipole moment, thus, all are rotationally active. Moreover, the non-zero dipole moment $(\mu)$ in all the three directions $\left(\mu_{a}, \mu_{\beta}, \mu_{\varsigma}\right)$ causes even more complexity in the rotational spectra. Notably, the dipole moment of the conformers of Leucine is comparatively lower than that of other proteinogenic amino acids investigated in the previous studies, ${ }^{33,59}$ mainly due to non-polar nature of the side chain substituent in Leucine, and also due to the reason that all the conformers relevant for inverting the chirality are oriented in 'cis' position than the more polarized 'trans' configuration as has been discussed in the previous work on Leucine. ${ }^{10}$

Furthermore, the Ray's asymmetry parameter, $x=(2 \mathrm{~B}-\mathrm{A}-\mathrm{C}) /(\mathrm{A}-\mathrm{C})$, is an indicative of the asymmetric nature of the species. All the species are in prolate range, either near-prolate symmetric top or prolate asymmetric top, with moment of inertia (I) related as $\mathrm{I}_{\mathrm{a}}<\mathrm{I}_{\mathrm{b}}{ }^{\sim} \mathrm{I}_{\mathrm{c}}$ along the three principal axis $(a, b, c)$. The species with $x$ value closer to -1 corresponds to the near-prolate symmetric top with extended configuration as in the case of conformers EQ0,$E$ EQ0, EQ0 $0_{I I I}$ as well as isomeric species EQ2 2 2a $, \mathrm{EQ}_{\mathrm{R} 3 \mathrm{a}}, \mathrm{EQ} 2_{\mathrm{R} 3}$, EQ1 $1_{\mathrm{R} 4}$. The greater the deviation from $x$ value of -1 , more is the asymmetric nature of the species. Besides this, the lowest value $x=-0.41$ for the conformer EQ0 IV signifies its most asymmetric nature and folded configuration due to the intermolecular hydrogen bonding interactions (as shown in Figure 1). The diverse values of the asymmetric parameter $\chi$ observed for the conformers and stereo-isomeric species of Leucine, as listed in Table 2, can be attributed to the branched form of side chain which causes variable extensions of overall molecular framework.

\subsubsection{Rotational Signatures in the Conformers of Leucine}

The different conformers of Leucine can be differentiated via their own unique rotational parameters listed in Table 2 and hence, having distinct rotational features. All the conformers except EQ0 II , because of relatively high value of dipole moment component $\mu_{\beta}$, can be characterised by their $b$-type transitions corresponding to selection rule: $\Delta K_{-1}= \pm 1, \pm 3, \pm 5, \ldots$, and $\Delta K_{+1}= \pm 1, \pm 3, \pm 5, \ldots$, where $K_{-1}$ and $K_{+1}$ are pseudo rotational quantum numbers defining the prolate and oblate type projections. ${ }^{58}$ The conformer EQ0 II , however, possesses higher dipole moment values along $a$ - and $c$-axis, thus, can be differentiated by $a$ -type transitions corresponding to $\Delta K_{-1}=0, \pm 2, \pm 4, \ldots$, and $\Delta K_{+1}= \pm 1, \pm 3, \pm 5, \ldots$, along with $c$-type transitions following $\Delta K_{-1}= \pm 1, \pm 3, \pm 5, \ldots$ and $\Delta K_{+1}=0, \pm 2, \pm 4, \ldots$, selection rules.

However, the value of principal rotational constant 'A' for conformers EQ0 II and EQ0 IV are nearly the same, thus, differentiating their rotational transitions can be quite strenuous. To resolve this complexity, two signatures can be followed. First is to locate their respective characteristic transitions, and second is the distinctly different values for nuclear quadrupole constants $\left(\chi_{\mathrm{aa}}, \chi_{\mathrm{bb}}, \chi_{\mathrm{cc}}\right)$. In fact, the marked differences 
in the values of the nuclear quadrupole constants, arising from the presence of nitrogen (with nuclear spin $\mathrm{I}=1 / 2$ ), is mainly due to differing electronic environment resulting from differently oriented substituents around the nitrogen in the two conformers. These distinct values result into different hyperfine splitting pattern which additionally acts as the characteristic rotational feature.

Another challenge is encountered in resolving the conformers EQ0 I and EQ0 $0_{I I I}$. The orientation of nitrogen is same in both besides a similar electronic environment due to the same surrounding atoms. However, EQ0 I can be differentiated by locating $c$-type transitions which are absent in EQ0 $0_{\text {III }}$ because of nearly zero value of dipole moment along the $c$-axis. But note that the value of dipole moment in $\mathrm{EQ0}$ I is also quite low, therefore, $c$-type transitions appear too weak. To additionally assist in differentiating these conformers, the associated isomeric species need to be followed as explored along the pathways discussed in stereoinversion of Leucine. ${ }^{10}$ From the pathways, it is quite apparent that the isomeric species, EQ1 $1_{\mathrm{R} 1}$ and EQ1 $1_{\mathrm{R} 2 \mathrm{~b}}$, can act as tracer for conformer $\mathrm{EQ}_{\mathrm{I}}$ whereas $\mathrm{EQ} 1_{\mathrm{R} 3 \mathrm{a}}$ and $\mathrm{EQ} 2_{\mathrm{R} 3}$ are related to conformer $\mathrm{EQ} 0_{\mathrm{III}}$.

\subsubsection{Rotational Signatures in the Isomeric Species of Leucine}

All the isomeric species located along the stereoinversion pathways of Leucine exhibit higher values of dipole moment and hence, exhibit more intense rotational transitions than the conformers of Leucine. Besides this, there is a significant variation in the value of rotational constants among these species, therefore, these can be uniquely identified. The zwitterionic ammonium ylide species, EQ1 $1_{\mathrm{R} 1}$, has the highest value of dipole moment followed by zwitterionic EQ1 $1_{\mathrm{R} 3 \mathrm{a}}$ and $\mathrm{EQ} 2_{\mathrm{R} 3}$ which can be characterised by stronger $b$ -type transitions in EQ1 $1_{\mathrm{R} 1}$ and $a$ - type transitions in EQ1 $1_{\mathrm{R} 3 \mathrm{a}}$ and $\mathrm{EQ} 2_{\mathrm{R} 3}$. The isomers $\mathrm{EQ} 1_{\mathrm{R} 3 \mathrm{a}}$ and $\mathrm{EQ} 22_{\mathrm{R} 3}$, despite being structurally the same, have entirely different rotational parameters, and hence can be distinctly characterised by their unique $(\mathrm{B}+\mathrm{C})$ transition line patterns associated with $a$-type transitions. ${ }^{58}$

Similarly, the imine-diol-type species, EQ1 $1_{\mathrm{R} 2 \mathrm{a}}, \mathrm{EQ} 2_{\mathrm{R} 2 \mathrm{a}}, \mathrm{EQ} 1_{\mathrm{R} 4}$, exhibit their own characteristic $a$-type transitions of quite high intensity. To the contrary, the en-diol species EQ1 $1_{\mathrm{R} 2 \mathrm{~b}}$ exhibits strongerb -type transitions. ${ }^{58}$ The spectral line database of significant ' $\mathrm{R}$ '-branch rotational transitions for all the species under study was further created using PGOPHER and is provided in SI Table S2 of supporting information. Note that in the present work, rotational transitions were considered only in the frequency range of 10000 $\mathrm{MHz}-45000 \mathrm{MHz}$ which are limited to the rotational quantum number, $J$ [?] 30. This frequency region actually belongs to higher intensity rotational transition lines shown in Figure 4, and is astrophysical quite important as discussed in the next section. Also note that the line-data in the higher frequency range of $34000 \mathrm{MHz}$ to $45000 \mathrm{MHz}$, which though is still lower than the typical astronomical observations, has also been provided in accordance to the highest frequency band 'one' of ALMA.

\subsection{Vibrational Signatures}

Leucine, a branched chain amino acid, and its isomeric species, all with 22 atoms, can exhibit sixty fundamental modes of vibrations in the mid- and far-infrared regions. In the present work, each mode has been characterised using a potential energy distribution (PED) analysis provided in SI Table S3. As discussed in the previous section on computational details, the DFT/B3LYP-D3(BJ)/cc-pVTZ method was found to be the optimum level for computing the vibrational data. To further examine the reliability of this quantum-mechanical method, the computed vibrational modes for global minimum EQ0 \# are compared with the experimentally available gas-phase vibrational data, ${ }^{30,31}$ as listed in Table 4 . The predicted gas-phase harmonic spectrum for EQ0\# is further depicted in Figure 3.

Note that Table 4 compares the vibrational modes computed for the gas-phase non-zwitterionic form of the global conformer of Leucine $\left(\mathrm{EQ}_{\#}\right)$ with those from the experimental matrix-isolation IR and fast thermal heating vibrational studies which are also associated with the neutral form of Leucine. The anharmonic calculations of the present work predict an intense -OH stretching vibration to appear at $3565 \mathrm{~cm}^{-1}$, which is in excellent agreement with the experimentally observed frequency listed in Table 4. The computed 'harmonic' frequencies though seem to be unreliable. The asymmetric and symmetric $-\mathrm{N}-\mathrm{H}$ stretching frequencies observed experimentally are also closely predicted by anharmonically computed frequencies at $3387 \mathrm{~cm}^{-1}$ and $3339 \mathrm{~cm}^{-1}$, respectively. Besides these, as listed in Table 4, the anharmonic $-\mathrm{C}-\mathrm{H}$ stretching 
frequencies corresponding to the modes, $\cup 4, \cup 5$ and $\cup 12$, are also computed within a reasonable agreement with the experimental values.

The intense characteristic $-\mathrm{C}=\mathrm{O}$ stretch in the global conformer was experimentally observed at $1768 \mathrm{~cm}^{-1}$ in a study by Stepanian et al., and at $1773 \mathrm{~cm}^{-1}$ by Linder et al., whereas the calculated harmonic frequency is at $1813 \mathrm{~cm}^{-1}$, however, its computed anharmonic frequency at $1783 \mathrm{~cm}^{-1}$ is in closer agreement with the experimental observations. Besides this, the bending vibrations including $-\mathrm{NH}_{2}$ bending, $-\mathrm{OH}$ and $\mathrm{CH}_{2}$ bending or even the torsional vibrations are also successfully predicted by the anharmonic calculations at B3LYP-D3(BJ)/cc-pVTZ level of theory as can be clearly seen in Table 4. However, the vibrational transitions in the far-infrared region are too complex to be characterized simply by observation. To resolve this complexity, potential energy distribution is a significant method to identify each mode successfully as has been additionally provided for global minimum EQ0\# in Table 4.

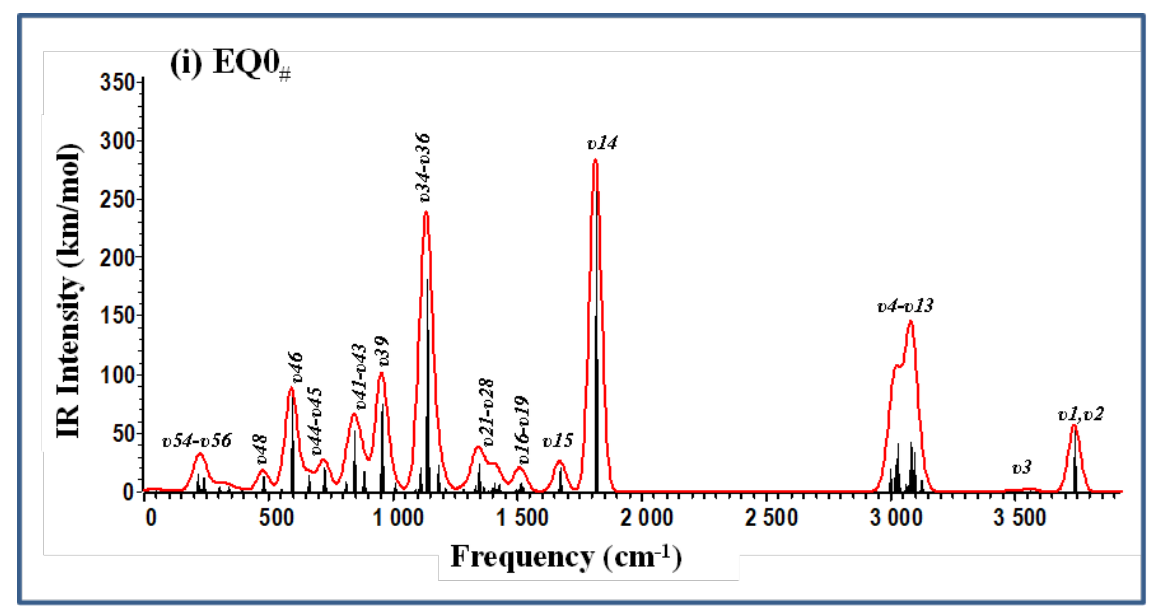

Figure 3. Vibrational spectrum (with unscaled harmonic frequencies), for global conformer EQ0\#, computed at B3LYP-D3(BJ)/cc-PVTZ level of the theory. The vertical solid lines (below the peaks) indicate normal modes of vibrations $(v)$, the details of which are provided in Table 4 and SI Table S3.

Table 4. Characteristic unscaled harmonic $(v$ harm $)$ and anharmonic $(v$ anharm $)$ frequencies (in $\left.\mathrm{cm}^{-1}\right)$ for global minimum conformer EQ0\# of Leucine, calculated at B3LYP-D3(BJ)/cc-pVTZ level of theory, at temperature of $298.15 \mathrm{~K}$. The experimental vibrational frequencies (indicated in bold) are taken from a study by Stepanian et al (Ref. 30), and those in italics are from a study by Linder et al (Ref. 31). For atom numbering in the vibrational assignments of different modes, refer to Figure 1. For the data on other species, see the supporting information.

(Assignment of modes, $v$ : stretching, $\beta$ : bending, $\tau$ : torsional, $\gamma$ : out of plane vibrations, asymm: asymmetric, sym: symmetric, + and - sign represents the relative direction of vibration in a mode)

\begin{tabular}{llllll}
\hline Mode & $v_{\text {harm }}$ & $v_{\text {anharm }}$ & Experimental $\left(v_{\exp }\right)$ & Experimental $\left(v_{\exp }\right)$ & Vibrational assignment $(P E D[?] 10 \%)$ \\
\hline & & & Ref. $[30]$ & Ref. $[31]$ & \\
$u 1$ & 3737 & 3565 & $\mathbf{3 5 6 5 . 7}$ & 3570 & $v\left[\mathrm{O}_{2}-\mathrm{H}_{22}\right]\{100\}$ \\
$\cup 2$ & 3562 & 3387 & $\mathbf{3 4 0 8 . 5}$ & & $v_{\text {asym }}\left[\mathrm{NH}_{2}\right]\{-98\}$ \\
$\cup 3$ & 3489 & 3339 & $\mathbf{3 3 3 9 . 5}$ & 2973 & $v$ symm $\left[\mathrm{NH}_{2}\right]\{98\}$ \\
$u 4$ & 3096 & 2944 & & 2942 & $v\left[\left(\mathrm{C}_{8}-\mathrm{H}_{16}\right)-\left(\mathrm{C}_{8}-\mathrm{H}_{18}\right)\right]\{89\}$ \\
$u 5$ & 3088 & 2941 & & 2889 & $v\left[\left(\mathrm{C}_{6}-\mathrm{H}_{12}\right)+\left(\mathrm{C}_{7}-\mathrm{H}_{13}\right)-\left(\mathrm{C}_{7}-\mathrm{H}_{14}\right)-\left(\mathrm{C}_{7}-\mathrm{H}_{15}\right)\right]\{-86$ \\
$u 12$ & 3017 & 2866 & & $v\left[\left(\mathrm{C}_{7}-\mathrm{H}_{13}\right)+\left(\mathrm{C}_{7}-\mathrm{H}_{14}\right)+\left(\mathrm{C}_{7}-\mathrm{H}_{15}\right)-\left(\mathrm{C}_{8}-\mathrm{H}_{17}\right)\right]\{78$ \\
$u 14$ & 1813 & 1783 & $\mathbf{1 7 6 8 . 2}$ & 1773 & $v\left[\mathrm{C}_{3}=\mathrm{O}_{1}\right]\{85\}$
\end{tabular}




\begin{tabular}{|c|c|c|c|c|c|}
\hline Mode & $v_{\text {harm }}$ & $v_{\text {anharm }}$ & Experimental $\left(v_{\exp }\right)$ & Experimental $\left(v_{\exp }\right)$ & Vibrational assignment (PED [?]10\%) \\
\hline u15 & 1670 & 1596 & 1641.3 & 1600 & $\beta\left[\mathrm{H}_{19}-\mathrm{N}_{9}-\mathrm{H}_{20}\right]\{-74\}$ \\
\hline$\cup 16$ & 1516 & 1465 & 1473.1 & 1466 & $\beta\left[\left(\mathrm{H}_{17}-\mathrm{C}_{8}-\mathrm{H}_{18}\right)-\left(\mathrm{H}_{16}-\mathrm{C}_{8}-\mathrm{H}_{18}\right)+\left(\mathrm{H}_{14}-\mathrm{C}_{7}-\mathrm{H}_{15}\right)-\right.$ \\
\hline$\cup 23$ & 1405 & 1362 & & 1363 & $\beta\left[\left(\mathrm{H}_{17}-\mathrm{C}_{8}-\mathrm{H}_{18}\right)+\left(\mathrm{H}_{16}-\mathrm{C}_{8}-\mathrm{H}_{18}\right)+\left(\mathrm{H}_{16}-\mathrm{C}_{8}-\mathrm{H}_{17}\right)-\right.$ \\
\hline ৩33 & 1144 & 1109 & 1123.7 & 1112 & $v\left[\left(\mathrm{C}_{5}-\mathrm{C}_{6}\right)-\left(\mathrm{C}_{6}-\mathrm{C}_{7}\right)+\left(\mathrm{C}_{6}-\mathrm{C}_{8}\right)\right]\{-12\}+v\left[\left(\mathrm{C}_{3}-\mathrm{C}_{4}\right)+\right.$ \\
\hline ט34 & 1123 & 1092 & 1104.1 & & $v\left[\left(\mathrm{C}_{4}-\mathrm{C}_{5}\right)+\left(\mathrm{C}_{3}-\mathrm{O}_{2}\right)-\left(\mathrm{N}_{9}-\mathrm{C}_{4}\right)\right]\{52\}$ \\
\hline ט38 & 961 & 927 & 924.1 & & $v\left[\left(\mathrm{C}_{3}-\mathrm{C}_{4}\right)-\left(\mathrm{C}_{5}-\mathrm{C}_{6}\right)+\left(\mathrm{C}_{6}-\mathrm{C}_{7}\right)-\left(\mathrm{C}_{4}-\mathrm{C}_{5}\right)-\left(\mathrm{N}_{9}-\mathrm{C}_{4}\right)\right]$ \\
\hline ט39 & 951 & 914 & 917.0 & & $\beta\left[\mathrm{H}_{19}-\mathrm{N}_{9}-\mathrm{C}_{4}\right]\{-11\}+\tau\left[\left(\mathrm{H}_{19}-\mathrm{N}_{9}-\mathrm{C}_{4}-\mathrm{C}_{5}\right)-\left(\mathrm{H}_{20^{-}} \mathrm{N}_{9^{-}}\right.\right.$ \\
\hline$\cup 41$ & 874 & 857 & 843.1 & & $v\left[\left(\mathrm{C}_{3}-\mathrm{C}_{4}\right)-\left(\mathrm{C}_{5}-\mathrm{C}_{6}\right)+\left(\mathrm{C}_{6}-\mathrm{C}_{7}\right)-\left(\mathrm{C}_{4}-\mathrm{C}_{5}\right)-\left(\mathrm{N}_{9}-\mathrm{C}_{4}\right)\right]$ \\
\hline$\cup 42$ & 856 & 829 & 834.3 & & $v\left[\left(\mathrm{C}_{5}-\mathrm{C}_{6}\right)+\left(\mathrm{C}_{6}-\mathrm{C}_{8}\right)+\left(\mathrm{N}_{9}-\mathrm{C}_{4}\right)\right]\{36\}+\tau\left[\left(\mathrm{H}_{19}-\mathrm{N}\right.\right.$ \\
\hline$\cup 43$ & 817 & 794 & 799.6 & & $v\left[\left(\mathrm{C}_{3}-\mathrm{C}_{4}\right)-\left(\mathrm{C}_{5}-\mathrm{C}_{6}\right)-\left(\mathrm{C}_{6}-\mathrm{C}_{7}\right)-\left(\mathrm{C}_{6}-\mathrm{C}_{8}\right)+\left(\mathrm{C}_{3}-\mathrm{O}_{2}\right)\right.$ \\
\hline$\cup 44$ & 746 & 722 & 725.2 & & $v\left[\left(\mathrm{C}_{5}-\mathrm{C}_{6}\right)+\left(\mathrm{C}_{6}-\mathrm{C}_{8}\right)+\left(\mathrm{N}_{9}-\mathrm{C}_{4}\right)\right]\{13\}+\gamma\left[\mathrm{O}_{1}-\mathrm{C}_{4}\right.$ \\
\hline$\cup 45$ & 654 & 648 & 646.5 & & $\beta\left[\mathrm{O}_{1}-\mathrm{C}_{3}-\mathrm{O}_{2}\right]\{56\}$ \\
\hline$\cup 46$ & 600 & 589 & 566.3 & & $\tau\left[\mathrm{H}_{22}-\mathrm{O}_{2}-\mathrm{C}_{3}-\mathrm{C}_{4}\right]\{85\}$ \\
\hline
\end{tabular}

Also note that in the previous study by Stepanian et al, only the scaled harmonic frequencies computed using B3LYP/aug-cc-pVTZ method were used to support the analysis from matrix-isolation IR stduy. ${ }^{30}$ However, the present study, employing the dispersion and anharmonic corrections at B3LYP/cc-pVTZ level of theory, successfully predicts the vibrational signatures without the use of any adhoc scaling factor (generally employed to harmonic values) as well as without using computationally demanding long range diffuse basis functions (as in aug-cc-pVTZ basis set). Moreover, in the present work, the anharmonic treatment of large amplitude vibrational motion associated with the intramolecular hydrogen bonding interactions in Leucine has also been carried out through VPT2, similar to the one employed in the case of Glutamic acid and Threonine. ${ }^{33,59}$ Note that due to an extended structural framework of Leucine and its isomeric species, all hydrogen bonding interactions span a distance over $1.6 \AA$ as evident in Figure 1, which is similar to the case of Glutamic acid. ${ }^{33}$ Thus, a closer agreement of anharmonically predicted frequencies with the experimental data for Leucine clearly reveals the efficacy of anharmonic approximation utilized in the present work. Therefore, the anharmonically calculated vibrational data in the present work for other isomeric species of Leucine, in the mid infrared and far-infrared frequency regions, is highly reliable, which is further provided in supporting information SI Table S3.

\section{ASTROPHYSICAL IMPORTANCE}

It is well-known from the physical and chemical traits of ISM that there is a continuous circulation of matter and energy at different stages of cosmic cycle in the ISM. Thus, there is a variation in the molecular density and temperature within the different regions of ISM. The low-temperature rotational line data computed in the present study can be utilized for searching the species in the cold regions of ISM while the computed vibrational spectroscopic data is quite reliable for search in the warmer star-forming regions of the ISM. In fact, these are the probable regions for observing various species being investigated: the neutral conformers of Leucine, zwitterionic ammonium ylide (EQ1 $\left.1_{\mathrm{R} 1}, \mathrm{EQ} 1_{\mathrm{R} 3 \mathrm{a}}, \mathrm{EQ} 2_{\mathrm{R} 3}\right)$, imine-diol (EQ1 $\left.1_{\mathrm{R} 2 \mathrm{a}}, \mathrm{EQ} 2_{\mathrm{R} 2 \mathrm{a}}, \mathrm{EQ} 1_{\mathrm{R} 4}\right)$, and ene-diol (EQ1 $\left.1_{\mathrm{R} 2 \mathrm{~b}}\right)$, similar to the case of Glutamic acid. ${ }^{33}$

Also note that in the present work, the rotational database for all the species is purposely created in lower frequency region than the frequencies utilized for the search of the simplest amino acid Glycine in the ISM. In all the astronomical searches so far for Glycine, for example, in the star forming regions SgrB2, Orion, and low mass protostar IRAS, the frequency region searched is greater than $80 \mathrm{MHz}$ and up to few GHz. ${ }^{60-62}$ However, a high moment of inertia associated with comparatively larger Leucine molecule and its isomeric species results into a comparatively lower value of rotational constants. ${ }^{45}$ Therefore, it is the lower frequency region where more intense rotational transitions for Leucine and its isomeric species can be observed. The lower frequency region becomes more important for search in dense cold molecular clouds where the temperature can be as low as $10 \mathrm{~K}$. To assist this, the rotational spectra simulated for EQ0\# with different values of rotational quantum number $J$ (20-80) at temperature of $10 \mathrm{~K}$ is further provided 
in Figure 4. It can be seen that the frequency range of $10000 \mathrm{MHz}$ to $45000 \mathrm{MHz}$ is the region of high intensity rotational transitions at $10 \mathrm{~K}$. The higher rotational levels though will also be get populated as in higher temperature zone but a large number of transitions from these will be too complex to resolve as can be concluded using values of rotational quantum numbers: $K_{\mathrm{a}}$ and $K_{\mathrm{c}}$ (with $-J[?] K_{\mathrm{a}}$ or $K_{\mathrm{c}}$ [?] $J$ ). Therefore, in the present study, the rotational transitions have been mainly analysed in the frequency range of $10000 \mathrm{MHz}$ to $45000 \mathrm{MHz}$ (with highest rotational state of $J=30$ ). Nevertheless, the lower frequency region of this range can act as important aid towards the laboratory assignment of the transitions whereas the higher range can directly assist the astronomical observations, for example, through Very Large Array Sky Survey (VLASS), futuristic lower frequency ALMA Band, and Square Kilometer Array (SKA). ${ }^{63-65}$

\section{Hosted file}

image4.emf available at https ://authorea.com/users/384438/articles/500049-quantum-mechanicalrotational-and-vibrational-signatures-of-astrophyscially-relevant-gas-phase-stereoisomeric-species-of-leucine

Figure 4. The simulated rotational spectra of $\mathrm{EQ} 0_{\#}$ for different $J$ values at a temperature of $10 \mathrm{~K}$.

Apart from the rotational region, the infrared region is used mainly for investigating the laboratory based interstellar ice compositions. The matrix-isolation IR experiments are quite challenging for analysing the gas phase spectral details of neutral molecules. Moreover, the lab-based IR spectra of gas-phase zwitterionic species can be even more difficult to observe. ${ }^{66}$ In such a case, the anharmonic vibrational spectral data provided in the present study can act as crucial aid to the laboratory based astrochemical studies. In fact, the search of zwitterionic type species among the presence of radicals and anions can help in unveiling a vast network of exotic molecular species in the ISM.

Moreover, in some astrochemical detections, the molecular species under investigation is not searched directly but through some related tracer species. This is particularly in the case of a molecular species having either quite low or zero dipole moment or having negligible induced polarization, for which the rotational and vibrational transitions are too weak to be observed. For example, in the typical case of $\mathrm{N}_{2}$, its protonated form $\mathrm{N}_{2} \mathrm{H}^{+}$was successfully rationalized in the ISM while utilizing the quantum-mechanically computed data, thereby, acting as a tracer for locating the $\mathrm{N}_{2} \cdot{ }^{67} \mathrm{In}$ a similar manner, the charged isomeric species $\left(\mathrm{EQ} 1_{\mathrm{R} 1}, \mathrm{EQ} 1_{\mathrm{R} 3 \mathrm{a}}, \mathrm{EQ} 2_{\mathrm{R} 3}\right)$ being investigated in the present work, which in fact have a high dipole moment, can act as tracer for the detection of corresponding conformers of Leucine. These conformers have low dipole moment, therefore, exhibit only weak rotational and vibrational transitions.

Furthermore, in the astronomical searches for Glycine, two important conformers having cis - $(\alpha-\mathrm{COOH})$ $\left\{\left(\mathrm{NH}_{2}\right) \mathrm{N}-\mathrm{HO}=\mathrm{C}\right\}$ and trans $-(\alpha-\mathrm{COOH})-\left\{\left(\mathrm{NH}_{2}\right) \mathrm{N}-\mathrm{H}-\mathrm{O}\right\}$ configuration are generally considered in the observations. ${ }^{68}$ This is due to an equal probability for the presence of these conformers irrespective of their relative thermal stability because of the peculiar environment of ISM. In fact, it is not prudent to presume that only a particular conformer will be present in extra-terrestrial environment. Therefore, in the present study, all the conformers of Leucine involved in its stereoinversion are investigated so as to assist their detection in the outer space. It should be noted that except the global minimum $\mathrm{EQ} 0_{\#}$, the studies on other conformers of Leucine are considerably lacking. Besides this, in the present work, the rotational parameters for transition state species connecting the isomeric species investigated in this work located along the stereoinversion pathways of Leucine have also been computed and additionally provided in SI Table S4 of supporting information. Although the detection of such transient species is highly unlikely but can be an exception in the exotic environment of ISM.

\section{CONCLUSIONS}

In this work, the rotational and vibrational data has been computed for the conformers of Leucine and various isomeric species located along the gas-phase stereoinversion pathways of Leucine. Notably, the isomeric species investigated exhibits diverse chemistry. For these species, the rotational parameters were quantum-mechanically calculated employing MP2 theory whereas the anharmonically corrected vibrational 
data was computed using DFT while incorporating the dispersion effects. The computed spectral data is found to be in excellent agreement with the available experimental data for the global conformer of Leucine. The rotational line data provided in this work in the frequency range of $10000 \mathrm{MHz}$ to $45000 \mathrm{MHz}$ though belongs only to the low frequency region but can be extended to any usable range utilizing the rotational parameters computed in the present work. A detailed vibrational analysis using PED calculations has also been provided for all the fundamental vibrational modes, in mid-infrared to far infrared regions. The spectroscopic data provided in this work can be quite reliable for assisting any search for amino acid Leucine in outer space.

\section{Supporting Information}

Supporting information (SI) includes Tables S1-S4.

Acknowledgments

Namrata Rani thanks University Grants Commission (UGC), New Delhi (India) for providing SRF(NET) fellowship. The authors are also grateful to the Department of Chemistry, Panjab University, Chandigarh, for providing other computational software and other resources.

Funding Information

Financial assistance from Science \& Engineering Research Board (SERB), India, under as research project (sanction order No. EMR/2016/002074) is gratefully acknowledged.

\section{Conflict of interest}

The authors declare no competing financial interest.

\section{Author Contributions}

Both authors contributed equally.

\section{References}

(1) Winnewisser, G.; Herbst, E.; Ungerechts, H. Spectroscopy Among The Stars. In Spectroscopy of the Earth's Atmosphere and Interstellar Medium ; Rao, K. N., Weber, A., Eds.; Academic Press: New York, 1993.

(2) McGuire, B. A. 2018 Census of Interstellar, Circumstellar, Extragalactic, Protoplanetary Disk, and Exoplanetary Molecules.Astrophys. J. Suppl. Ser. 2018 , 239 , 17(1-48).

(3) Dishoeck, E. F. V. Astrochemistry of Dust, Ice and Gas : Introduction and Overview. Faraday Discuss. $\mathbf{2 0 1 4 , 1 6 8 , 9 - 4 7 . ~}$

(4) Herbst, E. Three Milieux for Interstellar Chemistry: Gas, Dust, and Ice. Phys. Chem. Chem. Phys. 2014, $16,3344-3359$.

(5) Barlow, M. J.; Swinyard, B. M.; Owen, P. J.; Cernicharo, J.; Gomez, H. L.; Ivison, R. J.; Krause, O.; Lim, T. L.; Matsuura, M.; Miller, S.; et al. Detection of a Noble Gas Molecular Ion, ${ }^{36} \mathrm{ArH}^{+}$, in the Crab Nebula.Science. 2013, 342, 1343-1345.

(6) Güsten, R.; Wiesemeyer, H.; Neufeld, D.; Menten, K. M.; Graf, U. U.; Jacobs, K.; Klein, B.; Ricken, O.; Risacher, C.; Stutzki, J. Astrophysical Detection of the Helium Hydride Ion $\mathrm{HeH}^{+}$. Nature 2019 , 568 , 357-359.

(7) Loison, J. C.; Wakelam, V.; Hickson, K. M. The Interstellar Gas-Phase Chemistry of HCN and HNC. Mon. Not. R. Astron. Soc.2014, 443, 398-410.

(8) Thomas, E. Quantum Chemistry and Spectroscopy, 3rd ed.; Pearson: London, 2012.

(9) Fortenberry, R. C. Quantum Astrochemical Spectroscopy. Int. J. Quantum Chem. 2017, 117, 81-91. 
(10) Rani. N; Vikas. Extra-Terrestrial Gas-Phase Stereoinversion in Amino Acid Leucine: Thermal and Photochemical Channels. ChemPhysChem, 2020, 21, 1107-1118.

(11) Widicus Weaver, S. L. Millimeterwave and Submillimeterwave Laboratory Spectroscopy in Support of Observational Astronomy.Annu. Rev. Astron. Astrophys. 2019, 57, 79-112.

(12) Etim, E. E.; Arunan, E. Rotational Spectroscopy and Interstellar Molecules. J. Geophys. Res. Planets 2015, 5, 16-21.

(13) Carroll, B. W.; Ostlie, D. A.; Friedlander, M. Telescopes. InAn Introduction to Modern Astrophysics ; Cambridge University Press: Cambridge, UK, 2017.

(14) Van Dishoeck, E. F. ISO Spectroscopy of Gas and Dust: From Molecular Clouds to Protoplanetary Disks. Annu. Rev. Astron. Astrophys. 2004, 42 , 119-167.

(15) Hunter, G. L. K.; Brogden, W. B. Laboratory and Astronomical Detection of Cyclopropanylidene, $\mathrm{C}_{3} \mathrm{H}_{2}$.Astrophys. J. 1985 , 299 , L63-L66.

(16) Lee, T. J.; Bunge, A.; Schaefer, H. F. Toward the Laboratory Identification of Cyclopropenylidene. J. Am. Chem. Soc.1985, 107, 137-142.

(17) Bogey, M.; Demuynck, C.; Destombes, J. L. Laboratory Detection of the Protonated Carbon Dioxide by Submillimeter Wave Spectroscopy.Astron. Astrophys. 1984, 138, L11-L12.

(18) Thaddeus, P.; Guelin, M.; Linke, R. A. Three New "Nonterrestrial" Molecules. Astrophys. J. 1981 , 246 , L41-L45.

(19) Fortenberry, R. C.; Francisco, J. S. On the Detectability of the HSS, HSO, and HOS Radicals in the Interstellar Medium. Astrophys. J. 2017 , 835, 243(1-8).

(20) Fuente, A.; Goicoechea, J. R.; Pety, J.; Le Gal, R.; Martín-Doménech, R.; Gratier, P.; Guzmán, V.; Roueff, E.; Loison, J. C.; Muñoz Caro, G. M.; et al. First Detection of Interstellar $\mathrm{S}_{2} \mathrm{H}$. Astrophys. J. Lett. $\mathbf{2 0 1 7}, 851, \mathrm{~L} 49(1-6)$.

(21) Fortenberry, R. C.; Lee, T. J. Computational Vibrational Spectroscopy for the Detection of Molecules in Space. In Annual Reports in Computational Chemistry ; Elsevier B.V.: Amsterdam, 2019; Vol. 15, pp 173-202.

(22) Tielens, A. G. G. M. The Molecular Universe. Rev. Mod. Phys.2013 , 85 , 1021-1081.

(23) Van Dishoeck, E. F. Astrochemistry: Overview and Challenges. InProceedings of the International Astronomical Union; M. Cunningham, T. J. M. \& Y. A., Ed.; Cambridge, UK, 2017; Vol. 13, pp 1-20.

(24) Belloche, A.; Garrod, R. T.; Menten, K. M. Detection of a Branched Alkyl Molecule in the Interstellar Medium: Iso -Propyl Cyanide.Science. 2014 , 345 , 1584-1588.

(25) Mcguire, B. A.; Carroll, P. B.; Loomis, R. A.; Finneran, I. A.; Jewell, P. R.; Remijan, A. J.; Blake, G. A. Discovery of the Interstellar Chiral Molecule Propylene Oxide $\left(\mathrm{CH}_{3} \mathrm{CHCH}_{2} \mathrm{O}\right)$. Science. 2016 , 352 , 1449-1455.

(26) Ellinger, Y.; Pauzat, F.; Markovits, A.; Allaire, A.; Guillemin, J.-C. The Quest of Chirality in the Interstellar Medium. Astron. Astrophys. 2020 , 633 , A49(1-7).

(27) Cronin, J. R.; Pizzarello, S. Amino Acids in Meteorites. Adv. Sp. Res. 1983 , 3 , 5-18.

(28) Kwok, S. The Interstellar Medium. In Physics and Chemistry of the Interstellar Medium ; University Science Books: Sausalito, California, 2007.

(29) Cocinero, E. J.; Lesarri, A.; Grabow, J.; López, J. C.; Alonso, J. L. The Shape of Leucine in the Gas Phase. ChemPhysChem 2007 , 8 , 599-604. 
(30) Stepanian, S. G.; Yu, A.; Adamowicz, L. Conformational Composition of Neutral Leucine . Matrix Isolation Infrared and Ab Initio Study. Chem. Phys. 2013 , 423 , 20-29.

(31) Linder, R.; Nispel, M.; Häber, T.; Kleinermanns, K. Gas-Phase FT-IR-Spectra of Natural Amino Acids. Chem. Phys. Lett.2005, 409 , 260-264.

(32) Dokmaisrijan, S.; Sanghiran, V.; Nimmanpipug, P. The Gas Phase Conformers and Vibrational Spectra of Valine, Leucine and Isoleucine: An Ab Initio Study. J. Mol. Struct. THEOCHEM 2010 ,953 , 28-38.

(33) Rani, N.; Vikas. Rotational and Vibrational Analysis of Astrophysically Relevant Isomeric Species of Proteinogenic Glutamic Acid: A Quantum-Mechanical Computational Study. J. Mol. Spectrosc. 2020 , 369 , 111271(1-9).

(34) Moller, C.; Plesset, M. S. Note on an Approximation Treatment for Many-Electron Systems. Phys. Rev. 1934 , $46,618-622$.

(35) Burke, K.; Wagner, L. O. DFT in a Nutshell. Int. J. Quantum Chem. 2013 , 113 , 96-101.

(36) Ditchfield, R.; Hehre, W. J.; Pople, J. A. Self-Consistent Molecular-Orbital Methods. IX. An Extended Gaussian-Type Basis for Molecular-Orbital Studies of Organic Molecules. J. Chem. Phys.1971 , 54 , $724-728$.

(37) Lee, C.; Yang, W.; Parr, R. G. Development of the Colle-Salvetti Correlation-Energy Formula into a Functional of the Electron Density.Phys. Rev. B 1988, 37, 785-789.

(38) Becke, A. D. A New Mixing of Hartree-Fock and Local Density-Functional Theories. J. Chem. Phys. $1993,98,1372-1377$.

(39) Grimme, S.; Antony, J.; Ehrlich, S.; Krieg, H. Parametrization of Density Functional Dispersion Correction DFT-D for the 94 Elements H-Pu.J. Chem. Phys. 2010, 132 , 154104(1-19).

(40) Grimme, S.; Ehrlich, S.; Goerigk, L. Effect of the Damping Function in Dispersion Corrected Density Functional Theory. J. Comput. Chem. 2011, 32 , 1456-1465.

(41) Piccardo, M.; Penocchio, E.; Puzzarini, C.; Biczysko, M.; Barone, V. Semi-Experimental Equilibrium Structure Determinations by Employing B3LYP/SNSD Anharmonic Force Fields: Validation and Application to Semirigid Organic Molecules. J. Phys. Chem. A 2015 ,119 , 2058-2082.

(42) Goerigk, L.; Grimme, S. Double-Hybrid Density Functionals. Wiley Interdiscip. Rev. Comput. Mol. Sci. $2014,4,576-600$.

(43) Bloino, J.; Barone, V. A Second-Order Perturbation Theory Route to Vibrational Averages and Transition Properties of Molecules: General Formulation and Application to Infrared and Vibrational Circular Dichroism Spectroscopies. J. Chem. Phys. 2012 ,136, 124108(1-15).

(44) Barone, V.; Biczysko, M.; Bloino, J. Fully Anharmonic IR and Raman Spectra of Medium-Size Molecular Systems: Accuracy and Interpretation.Phys. Chem. Chem. Phys. 2014, 16, 1759-1787.

(45) Gordy, W.; L.Cook, R. Microwave Molecular Spectra, 3rd ed.; Weissberger, A., Ed.; John Wiley \& Sons: New York, 1984.

(46) Rao, K. N., Ed. Molecular Spectroscopy : Modern Research ; Academic Press: New York, 1985.

(47) Frisch, M. J.; G. W. Trucks, H. B. Schlegel, G. E. Scuseria, M. A. Robb, J. R. Cheeseman, G. Scalmani, V. Barone, B. Mennucci, G. A. Petersson, H. Nakatsuji, M. Caricato, X. Li, H. P. Hratchian, A. F. Izmaylov, J. Bloino, G. Zheng, J. L. Sonnenberg, M. Had, and D. J. F.; Frisch, M. J.; Trucks, G. W.; Schlegel, H. B.; Scuseria, G. E.; Robb, M. A.; Cheeseman, J. R.; Scalmani, G.; Barone, V.; et al. Gaussian 09, Revision D.01. Gaussian Inc., Wallingford . 2013. 
(48) Western, C. M. PGOPHER: A Program for Simulating Rotational, Vibrational and Electronic Spectra. J. Quant. Spectrosc. Radiat. Transf. 2017, 186, 221-242.

(49) Jamroz, M. H. Vibrational Energy Distribution Analysis VEDA 4. Warsaw 2004.

(50) Puzzarini, C.; Barone, V. A Never-Ending Story in the Sky: The Secrets of Chemical Evolution. Phys. Life Rev. 2019 ,32 , 59-94.

(51) Puzzarini, C.; Barone, V. The Challenging Playground of Astrochemistry: An Integrated Rotational Spectroscopy-Quantum Chemistry Strategy. Phys. Chem. Chem. Phys. 2020, 22, 6507-6523.

(52) Cernicharo, J.; Guelin, M.; Agundez, M.; McCarthy, M. C.; Thaddeus, P. Detection of $\mathrm{C}_{5} \mathrm{~N}$ - and Vibrationally Excited $\mathrm{C}_{6} \mathrm{H}$ in IRC +10216. Astrophys. J. 2008,688, L83-L86.

(53) Botschwina, P.; Oswald, R. Carbon Chains of Type $\mathrm{C}_{2 \mathrm{n}+1} \mathrm{~N}^{-}(\mathrm{N}=2-6)$ : A Theoretical Study of Potential Interstellar Anions. J. Chem. Phys. 2008 ,129, 044305(1-11).

(54) Guelin, M.; Green, S.; Thaddeus, P. Detection of the $\mathrm{C}_{4} \mathrm{H}$ Radical Toward IRC+10216. Astrophys. J.1978, 224, L27-L30.

(55) Cernicharo, J.; McCarthy, M. C.; Gottlieb, C. A.; Agundez, M.; Prieto, L. V.; Baraban, J. H.; Changala, P. B.; Guelin, M.; Kahane, C.; Martin-Drumel, M. A. et al. Discovery of SiCSi in IRC+10216: A Missing Link between Gas and Dust Carriers of Si-C Bonds. Astrophys. J. Lett. 2015, 806 , L3(1-6).

(56) Mccarthy, M. C.; Baraban, J. H.; Changala, P. B.; Stanton, J. F.; Martin-Drumel, M. A.; Thorwirth, S.; Gottlieb, C. A.; Reilly, N. J. Discovery of a Missing Link: Detection and Structure of the Elusive Disilicon Carbide Cluster. J. Phys. Chem. Lett. 2015 ,6 , 2107-2111.

(57) Puzzarini, C. Astronomical Complex Organic Molecules: Quantum Chemistry Meets Rotational Spectroscopy. Int. J. Quantum Chem.2017, 117, 129-138.

(58) Cooke, S. A.; Ohring, P. Decoding Pure Rotational Molecular Spectra for Asymmetric Molecules. J. Spectrosc. 2013 ,2013, 698392(1-10).

(59) Rani, N.;, Vikas. Computational rotational-vibrational spectroscopic analysis of isomeric species in the interstellar gas-phase stereoinversion of amino acid Threonine. Mol. Astrophys.2019, 15 , 8-16.

(60) Combes, F.; Rieu, Q. N.; Wlodarczak, G. Search for Interstellar Glycine. Astron. Astrophys. 1996 , $308,618-622$.

(61) Kuan, Y.; Charnley, S. B.; Huang, H.; Tseng, W.; Kisiel, Z. Interstellar Glycine. Astrophys. J. 2003 , $593,848-867$.

(62) Ceccarelli, C.; Loinard, L.; Castets, A.; Faure, A.; Lefloch, B. Search for Glycine in the Solar Type Protostar IRAS 16293-2422.Astron. Astrophys. 2000 , 362 , 1122-1126.

(63) Hiramatsu, M. ALMA and Its Observational Achievements: Unveiling the Mysteries of the Dark Universe. Fujitsu Sci. Tech. J.2017, 53, 9-14.

(64) Kellermann, K. I.; Bouton, E. N.; Brandt, S. S. Open Skies: The National Radio Astronomy Observatory and Its Impact on US Radio Astronomy. In Open Skies ; Springer: Cham, 2020; pp 319-390.

(65) Acosta-Pulido, J. A.; Agudo, I.; Alberdi, A.; Alcolea, J.; Alfaro, E. J.; Alonso-Herrero, A.; Anglada, G.; Arnalte-Mur, P.; Ascasibar, Y.; Ascaso, B.; et al. The Spanish Square Kilometre Array White Book ; Perez-Torres, M., Verdes-Montenegro, L., Guirado, J. C., Alberdi, A., Martin-Pintado, J., Eds.; Spain, 2015.

(66) Matrix Isolation Spectroscopy ; Barnes, A. J., Orville-Thomas, W. J., Muller, A., Gaufres, R., Eds.; D. Reidel Publishing Company: Dordrecht : Holland, 2007. 
(67) Qi, C.; Oberg, K. I.; Espaillat, C. C.; Robinson, C. E.; Andrews, S. M.; Wilner, D. J.; Blake, G. A.; Bergin, E. A.; Cleeves, L. I. Probing $\mathrm{CO}$ and $\mathrm{N}_{2}$ Snow Surfaces in Protoplanetary Disks with $\mathrm{N}_{2} \mathrm{H}^{+}$ Emission. Astrophys. J. 2019, 882, 160(1-19).

(68) Barone, V.; Biczysko, M.; Bloino, J.; Puzzarini, C. Accurate Structure, Thermodynamic and Spectroscopic Parameters from CC and CC/DFT Schemes: The Challenge of the Conformational Equilibrium in Glycine.Phys. Chem. Chem. Phys. 2013 , 15 , 10094-10111. 